PSS PROCEEDINGS

\title{
Three-loop heavy quark potential ${ }^{*}$
}

\author{
Alexander V. Smirnov \\ Scientific Research Computing Center of Moscow State University, Russia \\ E-mail: asmirnoverdm.ru
}

Vladimir A. Smirnov

Nuclear Physics Institute of Moscow State University, Russia

E-mail: smirnov@theory.sinp.msu.ru

\section{Matthias Steinhauser ${ }^{\dagger}$}

KIT

E-mail: matthias.steinhauser@kit.edu

We discuss the calculation of the three-loop corrections to the static potential of two heavy quarks.

35th International Conference of High Energy Physics

July 22-28, 2010

Paris, France

*This work is supported by DFG through SFB/TR 9 "Computational Particle Physics" and RFBR, grant 08-0201451. Preprint Nos.: SFB/CPP-10-82, TTP10-36

†peaker. 


\section{Introduction}

The potential between two heavy quarks has been among the first application after the formulation of QCD. At leading order it is given by the exchange of a Coulomb gluon and can - after obvious modifications - be obtained from the potential of the hydrogen atom. One- and two-loop corrections have been considered in Refs. $[1,2,3,4,5,6]$ and have introduced numerically sizable effects in quarkonium physics (see, e.g., the review Ref. [7]). Since 1998 there has been a raising interest in the three-loop corrections. The fermionic corrections have been completed in 2008 [8] and in 2009 two independent groups $[9,10]$ have completed the purely abelian three-loop part. In Refs. [8,9] the calculation has been performed in a covariant gauge and the independence of the final expression on the gauge parameter has been a crucial check for the correctness of the result.

\section{Outline of the calculation}

The calculation of the static potential requires the evaluation of the four-point amplitude of a heavy quark and anti-quark. Some sample Feynman diagrams are shown in Fig. 1. It is sufficient to consider as a starting point the so-called non-relativistic QCD (NRQCD), i.e. QCD with hard degrees of freedom integrated out. In this limit the heavy quark propagators represent static colour sources with propagators $1 / p_{0}$ whereas the gluons and light quarks are still relativistic. The only dimensionful scale in the problem is the momentum transfer between the heavy quark and anti-quark and thus momentum integrals can be represented by two-point functions. In Fig. 2 the different cases of the scalar two-point integrals up to three loops are shown.

In case the static lines are absent the problem of computing the corresponding integrals up to three loops has been solved many years ago [11] and a public code exists, MINCER [12] which can easily be included in all computational frameworks. The presence of the static lines, however, makes the practical evaluation quite difficult and an explicit solution of the recurrence problem (as implemented in Ref. [12]) is not available. Furthermore, the master integrals are significantly more complicated due to the occurrence of the static lines.

In Refs. [8,9] the reduction of all occurring integrals to a small set of master integrals has been achieved with the help of the program FIRE [13] which can be linked to a database and thus handle non-trivial problems in a quite efficient way. In our case up to 16 indices have to be
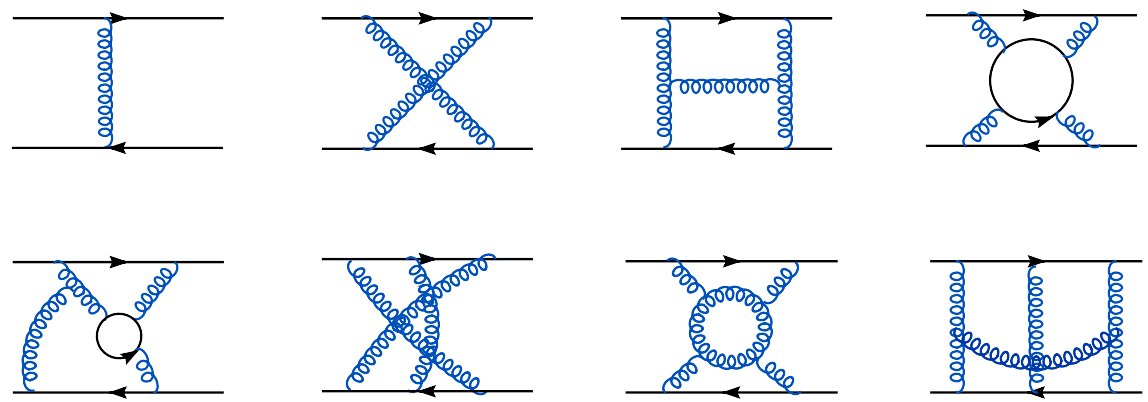

Figure 1: Sample diagrams contributing to the static potential at tree-level, one-, two- and three-loop order. Solid and curly lines represent quarks and gluons, respectively. In the case of closed loops the quarks are massless; the external quarks are heavy and treated in the static limit. 


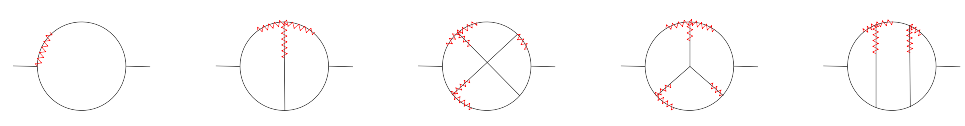

Figure 2: Scalar one-, two- and three-loop diagrams. The solid line stands for massless relativistic propagators and the zigzag line represents static propagators.

considered: $8+6=14$ indices from relativistiv and non-relativistic propagators, respectively, and in addition one index from an irreducible numerator (see Fig. 2). The problem can be simplified by considering a partial fractioning in those cases where three static lines meet in one vertex and by linear relations between four static propagators which leads to at most three static propagators at three loops thus reducing the total number of indices to twelve. We have performed the calculation in both ways. In the first option with up to 15 indices only little manual work is involved, however, significantly more computer resources are needed than in the twelve-index approach. In the latter case one has to provide several relations implemeting the partial fractioning (mentioned above) and symmetry relations to end up with a small set of different case to be considered for the reduction. The fact that the final results in both approaches agree constitutes a strong check on our result.

After the reduction one ends up with 41 master integrals for which an explicit result is needed. Nine integrals are quite simple and can essentially be obtained from the one- and two-loop results. 14 integrals contain a massless one-loop diagram which can be integrated out leading to a two-loop integral with an exponent depending on the space-time dimension $d$. These integrals are already quite involved and have been presented in Ref. [14]. The remaining 18 integrals are genuinely of three-loop order and involve a nontrivial calculation to obtain their result. All but three $\varepsilon=$ $(4-d) / 2$ coefficients could be computed analytically; the corresponding analytical results have been presented in Ref. [15]. The three missing coefficients are known with a numerically precision sufficient for all foreseeable applications.

\section{Static potential to three loops}

Let us finally present the result for the static potential. We refrain from analytical results which can be found in Refs. [8, 9] but immediately show $V(|\vec{q}|)$ in numerical from:

$$
\begin{aligned}
V(|\vec{q}|)= & -\frac{4 \pi C_{F} \alpha_{s}(|\vec{q}|)}{\vec{q}^{2}}\left[1+\frac{\alpha_{s}}{\pi}\left(2.5833-0.2778 n_{l}\right)+\left(\frac{\alpha_{s}}{\pi}\right)^{2}\left(28.5468-4.1471 n_{l}+0.0772 n_{l}^{2}\right)\right. \\
& \left.+\left(\frac{\alpha_{s}}{\pi}\right)^{3}\left(209.884(1)-51.4048 n_{l}+2.9061 n_{l}^{2}-0.0214 n_{l}^{3}\right)+\cdots\right],
\end{aligned}
$$

where $\mu^{2}=\vec{q}^{2}$ has been adopted in order to suppress the infrared logarithm and the ellipses denote higher order terms in $\alpha_{s}$. It is interesting to note that the term "209" in the three-loop coefficient receives a large contribution ("211") from the term with colour factor $C_{A}^{3}$ whereas the new colour structure $d_{F}^{a b c d} d_{A}^{a b c d}$ only contributes with a coefficient "- 2 ". From Eq. (3.1) we observe at one-, two- and three-loop order a large screening of the non-fermionic contributions by the $n_{l}$ terms which is most prominent in the three-loop coefficient for $n_{l}=5$.

In Tab. 1 we show the numerical evaluation of the square bracket of Eq. (3.1) for the charm, bottom and top quark case, i.e. for $n_{l}=3,4$ and 5, adopting the appropriate values of $\alpha_{s}$. For 


\begin{tabular}{c|l|l|l|l}
$n_{l}$ & $\alpha_{s}^{\left(n_{l}\right)}$ & 1 loop & 2 loop & 3 loop \\
\hline 3 & 0.40 & 0.2228 & 0.2723 & 0.1677 \\
4 & 0.25 & 0.1172 & 0.08354 & 0.02489 \\
5 & 0.15 & 0.05703 & 0.02220 & 0.002485
\end{tabular}

Table 1: Radiative corrections to the potential $V(|\vec{q}|)$ where the tree-level result is normalized to 1 . In the second column we also provide the numerical value of $\alpha_{s}$ corresponding to the soft scale where $\mu \approx m_{q} \alpha_{s}$ ( $m_{q}$ being the heavy quark mass).

charm the three-loop corrections are almost as big as the one- and two-loop contributions whereas for bottom the three-loop contribution is already a factor of four smaller than the two-loop one. In the case of the top quark one observes a good convergence: the three-loop term is already a factor ten smaller than the two-loop counterpart.

To summarize, the three-loop corrections to the static heavy quark potential are available and can now be used for the prediction of the top quark threshold production at a future linear collider with third-order accuracy, for the precise extraction of the bottom quark mass from $\Upsilon$ sum rules, and for the comparison of the potential with results obtained on the lattice in order to gain insight to the validity of perturbation theory.

\section{References}

[1] W. Fischler, Nucl. Phys. B 129 (1977) 157.

[2] A. Billoire, Phys. Lett. B 92 (1980) 343.

[3] M. Peter, Phys. Rev. Lett. 78 (1997) 602 [arXiv:hep-ph/9610209].

[4] M. Peter, Nucl. Phys. B 501 (1997) 471 [arXiv:hep-ph/9702245].

[5] Y. Schroder, Phys. Lett. B 447 (1999) 321 [arXiv:hep-ph/9812205].

[6] B. A. Kniehl, A. A. Penin, M. Steinhauser and V. A. Smirnov, Phys. Rev. D 65 (2002) 091503 [arXiv:hep-ph/0106135].

[7] N. Brambilla et al. [Quarkonium Working Group], arXiv:hep-ph/0412158.

[8] A. V. Smirnov, V. A. Smirnov and M. Steinhauser, Phys. Lett. B 668 (2008) 293 [arXiv:0809.1927 [hep-ph]].

[9] A. V. Smirnov, V. A. Smirnov and M. Steinhauser, Phys. Rev. Lett. 104 (2010) 112002 [arXiv:0911.4742 [hep-ph]].

[10] C. Anzai, Y. Kiyo and Y. Sumino, Phys. Rev. Lett. 104 (2010) 112003 [arXiv:0911.4335 [hep-ph]].

[11] K. G. Chetyrkin and F. V. Tkachov, Nucl. Phys. B 192 (1981) 159.

[12] S. A. Larin, F. V. Tkachov and J. A. M. Vermaseren, "The Form Version Of Mincer."

[13] A. V. Smirnov, JHEP 0810 (2008) 107 [arXiv:0807.3243 [hep-ph]].

[14] A. V. Smirnov, V. A. Smirnov and M. Steinhauser, arXiv:1006.5513 [hep-ph].

[15] A. V. Smirnov, V. A. Smirnov and M. Steinhauser, PoS RADCOR2009 (2010) 075 [arXiv:1001.2668 [hep-ph]]. 